\title{
Phase 3 Randomized 3-Month Trial with an Ongoing 3-Month Safety Extension of Fixed-Combination Brinzolamide 1\%/Brimonidine 0.2\%
}

\author{
Quang H. Nguyen, ${ }^{1}$ Matthew G. McMenemy, ${ }^{2}$ Tony Realini, ${ }^{3}$ Jess T. Whitson, ${ }^{4}$ and Stephen M. Goode ${ }^{5}$
}

\begin{abstract}
Purpose: This study compared the intraocular pressure (IOP)-lowering efficacy of fixed-combination brinzolamide $1 \%$ /brimonidine $0.2 \%$ (BBFC) with that of its component medications, brinzolamide and brimonidine, in patients with open-angle glaucoma or ocular hypertension.

Patients and Methods: In this phase 3, multicenter, double-masked, parallel-group, 3-month study with a 3-month safety extension, eligible patients were randomized 1:1:1 to treatment with BBFC, brinzolamide, or brimonidine thrice daily after a washout period, during which any IOP-lowering medications were discontinued. The primary objectives of this study were to determine whether the IOP-lowering efficacy of BBFC was superior to that of brinzolamide alone and, separately, of brimonidine alone. IOP was assessed at 8:00 AM, 10:00 AM, 3:00 PM, and 5:00 PM at 2 weeks, 6 weeks, and 3 months after study drug initiation.

Results: A total of 690 patients were enrolled in the study, and 615 completed the 3-month visit. Baseline mean IOP levels were similar among the 3 treatment groups at each of the 4 time points assessed. At the 3-month primary endpoint, mean IOP of the BBFC group was significantly lower than that of either the brinzolamide group or the brimonidine group $(P \leq 0.005)$ across all time points. At the 2 - and 6-week supportive endpoints, mean IOP of the BBFC group was significantly lower at all time points than the mean IOP of either the brinzolamide group $(P \leq 0.01)$ or the brimonidine group $(P<0.0001)$. A total of 143 patients experienced at least 1 treatment-related adverse event (AE; BBFC group, $n=58,26.2 \%$; brinzolamide group, $n=44,18.8 \%$; brimonidine group, $n=41,17.4 \%$ ), the majority of which were ocular AEs.

Conclusions: This study demonstrated that BBFC has significantly superior IOP-lowering activity compared with either brinzolamide $1 \%$ or brimonidine $0.2 \%$ in patients with open-angle glaucoma or ocular hypertension while providing a safety profile which is consistent with that of the individual components.
\end{abstract}

\section{Introduction}

$\mathbf{R}$ EDUCTION IN INTRAOCULAR PRESSURE (IOP) has been shown to decrease the risk of glaucomatous progression for patients with open-angle glaucoma, ${ }^{1-3}$ and to reduce the risk of the development of glaucoma in patients with ocular hypertension. ${ }^{4,5}$ However, $40 \%$ of patients on monotherapy fail to reach an acceptable target IOP and require treatment with 2 or more antihypertensive medications, and nearly $10 \%$ require 3 or more, to achieve a modest $20 \%$ IOP reduction. ${ }^{4}$ Two antihypertensive medications can be administered concomitantly or as a single fixed-combination therapy. Several fixed-combination therapies have been developed for use in open-angle glaucoma, with each therapy adding a second drug class to a beta antagonist. Each fixedcombination therapy combines the beta antagonist timolol $0.5 \%$ with one of the following drug classes: a carbonic anhydrase inhibitor (CAI; dorzolamide $2 \%$ /timolol $0.5 \%$ and brinzolamide $1 \%$ /timolol $0.5 \%$ ), a prostaglandin analog (PGA; latanoprost $0.005 \%$ / timolol $0.5 \%$, travoprost $0.004 \%$ / timolol $0.5 \%$, and bimatoprost $0.03 \% /$ timolol $0.5 \%$ ), or an alpha agonist (brimonidine $0.2 \%$ /timolol 0.5\%).

Fixed-combination therapies hold several advantages over the concurrent administration of 2 distinct medications,

\footnotetext{
${ }^{1}$ Scripps Clinic, La Jolla, California.

${ }^{2}$ Lone Star Eye Care, Sugar Land, Texas.

${ }^{3}$ West Virginia University Eye Institute, Morgantown, West Virginia.

${ }^{4}$ University of Texas Southwestern Medical Center at Dallas, Dallas, Texas.

${ }^{5}$ Alcon Research, Ltd., Fort Worth, Texas.
} 
including the avoidance of the potential for washout of the first drug by the second, ${ }^{6}$ the patient convenience of having only 1 medication bottle and a reduced number of eye drops to dispense, potentially lower cost as a result of fewer copays, and the reduction in ocular exposure to preservatives, which have been implicated in the development of ocular surface disease in patients with glaucoma. ${ }^{7,8}$ However, all fixed-combination IOP-lowering therapies currently available contain timolol, which is contraindicated in patients with certain respiratory or cardiac conditions, such as asthma, severe chronic obstructive pulmonary disease, sinus bradycardia, second- or third-degree atrioventricular block, cardiogenic shock, or overt cardiac failure. ${ }^{9}$

A new fixed combination has been developed that combines a CAI with an alpha agonist: brinzolamide $1 \% /$ brimonidine $0.2 \%$ (BBFC). Both brinzolamide and brimonidine have been used as a part of other fixed-combination therapies (with timolol), and both have shown clinically relevant IOP reductions when used adjunctively with a PGA. ${ }^{10-12}$ BBFC, which contains only $0.003 \%$ benzalkonium chloride, represents a fixed-combination option for patients with contraindications to beta antagonists. The current study used a randomized design to measure the contribution of the individual active ingredients of BBFC and to assess its safety. The aim of this study was to compare the IOP-lowering effect of BBFC with that of its component medications (brinzolamide $1 \%$ and brimonidine $0.2 \%$ ) in patients with open-angle glaucoma or ocular hypertension.

\section{Methods}

\section{Study design}

This was a phase 3 , randomized, multicenter, doublemasked, parallel-group, 3-month, 3-arm contribution-ofelements study with a 3-month safety extension in patients with open-angle glaucoma or ocular hypertension. Data from the first 3 months are presented here; data from the safety extension will be presented in another article. The protocol was approved by all appropriate institutional review boards, and the study was performed in compliance with the ethical principles of the Declaration of Helsinki and Good Clinical Practice. All participating patients provided written informed consent before initiation of any screening, washout, or other study-related procedures.

\section{Participants}

Patients from 65 academic and private practice study sites throughout the United States were recruited by investigators to participate in this study. Eligibility criteria are shown in Table 1.

\section{Schedule of visits and assessments}

At the screening visit, patients were screened against the inclusion and exclusion criteria. Eligible patients were then instructed to discontinue use of all IOP-lowering medications during a washout period (at least 5 days for miotics and oral/topical CAIs, 14 days for alpha agonists and alpha/beta agonists, and 28 days for beta antagonists and PGAs). For combination drugs, the longest washout period of the individual components was used. Patients were permitted, at the investigator's discretion, to substitute a medication with a shorter washout period.
The first eligibility visit was scheduled at the end of the washout period for those patients on prior IOP-lowering medications and $3 \pm 1$ days for those patients not taking any IOP-lowering medications. At this visit, investigators assessed patient IOP in both eyes at 8:00 AM, 10:00 AM, 3:00 $\mathrm{PM}$, and 5:00 PM, taking the mean of 2 values at each time point, or the mean of the closest 2 of 3 values if the first 2 values differed by $>4 \mathrm{~mm} \mathrm{Hg}$. At the 8:00 AM time point, they also performed best-corrected visual acuity (BCVA) and slit-lamp biomicroscopy on both eyes, and at both the 8:00 $\mathrm{AM}$ and 10:00 AM time points, they measured resting pulse rate and blood pressure. At the second eligibility visit, which was scheduled 3-8 days after the first eligibility visit, investigators assessed the same parameters as during the first eligibility visit (IOP, BCVA, slit-lamp biomicroscopy, and resting pulse rate and blood pressure) on the same schedule. At both eligibility visits, patients had to have study eye mean IOP between 24 and $36 \mathrm{~mm} \mathrm{Hg}$ at the 8:00 AM time point and between 21 and $36 \mathrm{~mm} \mathrm{Hg}$ at the 10:00 AM time point, as well as an IOP $36 \mathrm{~mm} \mathrm{Hg}$ or less in both eyes at all time points. All eligible patients were then randomized 1:1:1 to treatment with BBFC, brinzolamide $1 \%$, or brimonidine $0.2 \%$. The randomization was stratified by the mean baseline IOP from both eligibility visits measured at the 8:00 AM time point (24-27 and $28-36 \mathrm{~mm} \mathrm{Hg}$ ) to ensure balanced baseline IOP among treatment groups. Patients dosed both eyes with study medications (unless the non-study eye had a potential safety issue) thrice daily (TID) at 8:00 AM ( $\pm 30 \mathrm{~min}), 3: 00$ PM $( \pm 30 \mathrm{~min})$, and 10:00 PM $( \pm 30 \mathrm{~min})$ for 6 months.

Patients returned for visits 2 weeks, 6 weeks, 3 months, and 6 months after the second eligibility visit. Patients did not instill the 8:00 AM dose of study medication before these visits. At these visits, investigators assessed patient IOP in both eyes at 8:00 AM (15 min after which investigators administered study medication), 10:00 AM, 3:00 PM (15 min after which investigators administered study medication), and 5:00 PM. At the 8:00 AM time point, they also performed BCVA and slit-lamp biomicroscopy on both eyes, and at both the 8:00 $\mathrm{AM}$ and 10:00 AM time points, they measured resting pulse rate and blood pressure. In addition, at the 3- and 6-month visits, investigators performed automated perimetry in both eyes at 8:00 AM and dilated fundus examination and pachymetry at 5:00 PM in both eyes.

\section{Outcomes}

Efficacy assessments were composed of IOP evaluations, which were performed using a Goldmann applanation tonometer. The primary efficacy parameter was the mean IOP at the 3-month visit at each of the 4 time points. The secondary efficacy parameter was the mean IOP at the 2- and 6-week visits for all time points.

Safety assessments were composed of solicited and unsolicited adverse events (AEs), BCVA, slit-lamp biomicroscopy observations, pachymetry, automated perimetry, fundus parameters, and resting pulse rate and blood pressure, all collected through the 3-month visit. Additional safety data from the 6-month visit will be analyzed separately once that information is available. AEs were collected, monitored, and evaluated throughout the study and were recorded at each visit. Slit-lamp biomicroscopy included examination of the cornea, lens, eyelids, conjunctiva, iris, and anterior chamber of both eyes. Dilated fundus examination 
Table 1. Inclusion and Exclusion CRiteria for the Study

Administrative and demographic requirements: patients

- were required to be willing and able to provide informed consent before screening;

- were required to be at least 18 years of age;

- could be of either gender and any race/ethnicity; and

- could not be of childbearing potential (unless they were abstinent or using a highly effective birth control measure), pregnant or intending to become pregnant during the study, or lactating.

Ocular requirements: patients were required to have

- a clinical diagnosis of open-angle glaucoma or ocular hypertension in at least 1 (study) eye;

- a mean baseline IOP in the study eye that was, at both eligibility visits,

- between 24 and $36 \mathrm{~mm} \mathrm{Hg}$ at the 8:00 AM time point,

- between 21 and $36 \mathrm{~mm} \mathrm{Hg}$ at the 10:00 AM time point; and

- an IOP $\leq 36 \mathrm{~mm} \mathrm{Hg}$ in both eyes at all time points of both eligibility visits.

Exclusion criteria, related to the eye: patients could not have

- any history of

- ocular trauma or intraocular surgery within the past 6 months; or

- ocular infection, inflammation, or laser surgery within the past 3 months; or

- any present observation of

$\circ$ any form of glaucoma other than open-angle glaucoma;

- chronic, recurrent, or severe inflammatory eye disease;

- central cornea thickness $>620 \mu \mathrm{m}$, as measured by pachymetry, in either eye;

- Shaffer angle grade $<2$ in either eye (range, 0 [complete or partial closure] to 3 [wide open angle, $>20^{\circ}$ ]), as measured by gonioscopy;

- cup/disc ratio >0.80 (horizontal or vertical measurement) in either eye;

- severe central visual field loss in either eye;

- clinically significant or progressive retinal disease;

- best-corrected visual acuity worse than $0.6 \log$ MAR;

- other ocular pathology (including severe dry eye) that may preclude the administration of an alpha-adrenergic agonist and/or a topical CAI;

- any abnormality preventing reliable applanation tonometry; or

- an inability to safely discontinue use of or go without all IOP-lowering ocular medication(s) for the specified washout period.

Exclusion criteria, related to systemic health: patients could not

- have a recent history of using study-prohibited medications, including

$\circ$ another investigational agent within 30 days before the screening visit;

- high-dose ( $>1 \mathrm{~g}$ daily) salicylate therapy within 4 weeks of the first eligibility visit; or

- any medications or substances used on a chronic basis that may affect IOP and which had not been on a stable dosing regimen for at least 30 days before the screening visit;

- use any prohibited medications during the study, including

- current or anticipated treatment with any psychotropic drugs that augment an adrenergic response;

- concurrent use of a monoamine oxidase inhibitor; or

- use of any additional topical or systemic ocular hypotensive medication during the study;

- have any other conditions that would make the patient unsuitable for the study, including

- history of active, severe, unstable, or uncontrolled systemic disease precluding safe administration of a topical alpha-adrenergic agonist or CAI; or

- hypersensitivity to alpha-adrenergic agonist drugs, topical or oral CAIs, sulfonamide derivatives, or any component of the study medications; or

- any condition that could require treatment with glucocorticoids, unless they could be safely discontinued during the study.

IOP, intraocular pressure; CAI, carbonic anhydrase inhibitor.

included examination of vitreous, retina, macula, choroid, and optic nerve (including cup/disc ratio) of both eyes. Blood pressure was also monitored.

\section{Objectives}

The primary objective of this study was to determine whether the IOP-lowering efficacy of BBFC, when dosed TID at 8:00 AM, 3:00 PM, and 10:00 PM, was superior to that of brinzolamide and, separately, was superior to that of brimonidine in patients with open-angle glaucoma or ocular hypertension after 3 months of treatment. We hypothesized that, on average, patients with open-angle glaucoma or ocular hypertension who were treated with BBFC for 6 months would demonstrate, at all 4 time points of the 3-month visit, a significantly lower mean IOP than that of patients treated with either brinzolamide alone or brimonidine alone.

\section{Statistical methods}

Pairwise comparisons of mean IOP (BBFC vs. brinzolamide and BBFC vs. brimonidine) at each scheduled ontherapy study visit at all 4 time points (8:00 AM, 10:00 AM, 3:00 PM, and 5:00 PM) were based on the least-squares means derived from a statistical model that accounts for correlated IOP measurements within patients, and were made using paired $t$-tests. Assuming a standard deviation between 3.5 and $3.9 \mathrm{~mm} \mathrm{Hg}$, this study provided a $90 \%$ 
power to detect a difference of at least $1.5 \mathrm{~mm} \mathrm{Hg}$ between any 2 treatment groups if at least 143 patients were analyzed for each treatment group. Target enrollment was 250 patients for each treatment group, with the intention of retaining no $<200$ patients per treatment group for safety assessment at the 3-month visit and 100 patients per treatment group for safety assessment at the 6-month visit.

An $\alpha$-level of 0.05 was used to declare statistical significance. All data analyses, which were intent-to-treat (ITT), were 2 sided. Data were analyzed for the baseline visit and the 3-month visit (primary efficacy endpoint, interim safety endpoint). Descriptive statistics were calculated for all safety parameters (AEs, BCVA, slit-lamp biomicroscopy observations, pachymetry, automated perimetry, fundus parameters, and resting pulse rate and blood pressure) and for IOP, IOP change from baseline, and IOP percent change from baseline. If both eyes were eligible, both were treated with study medication, but only the eye with the higher IOP at 8:00 AM that averaged across the 2 eligibility visits was selected for analysis. If both eyes had equal IOPs at 8:00 AM, the eye with the higher IOP at 10:00 AM that averaged across the 2 eligibility visits was selected for analysis. If both eyes had equal IOP at both time points, the right eye was selected for analysis. The last observation carried forward was employed for any missing IOP data points at 6 weeks and at 3 months. Statistical analysis was performed using SAS (SAS Institute, Cary, NC).

\section{Results}

\section{Participant flow}

A total of 690 patients were enrolled in the study, 679 were included in the ITT population, and 615 completed the study through the 3-month visit.

\section{Demographics and baseline characteristics}

Demographic and baseline characteristics were well balanced among the 3 arms (Table 2). Mean age of the ITT population was $64.9 \pm 10.4$ years, $77.9 \%$ of the population was white, and $56.1 \%$ were women.

\section{Intraocular pressure}

Baseline mean IOP levels were similar among the 3 treatment groups at each of the 4 time points. For the 3-month primary endpoint, mean IOP of the BBFC group was significantly lower than that of either the brinzolamide group or the brimonidine group $(P \leq 0.005$; Table 3$)$. For the 2- and 6-week supportive endpoints, mean IOP of the BBFC group was significantly lower at all time points than the mean IOP of either the brinzolamide group $(P \leq 0.01)$ or the brimonidine group $(P<0.0001 ; 6$-week data not shown). At the 3-month visit, the IOP superiority observed in the BBFC group ranged from a $3.7 \%$ improvement to a $13.4 \%$ improvement in \% IOP reduction from baseline (Fig. 1). These reductions in the BBFC group ranged from 5.4 to $8.4 \mathrm{~mm} \mathrm{Hg}$ (across time points), from 4.2 to $5.7 \mathrm{~mm} \mathrm{Hg}$ in the brinzolamide group, and from 3.1 to $6.5 \mathrm{~mm} \mathrm{Hg}$ in the brimonidine group (data not shown).

\section{AEs and other safety measures}

A total of 143 patients experienced at least 1 treatmentrelated $\mathrm{AE}$ (BBFC group, $n=58,26.2 \%$; brinzolamide group, $n=44,18.8 \%$; brimonidine group, $n=41,17.4 \%$ ), most of which were ocular AEs (Table 4). The brinzolamide-containing groups experienced all cases of blurred vision (BBFC group, $4.5 \%$; brinzolamide group, 6.8\%) and eye pruritus (BBFC group, $2.3 \%$; brinzolamide group, $1.3 \%$ ) and nearly all cases of dysgeusia (BBFC group, 4.1\%; brinzolamide group, 10.3\%; brimonidine group, $0.4 \%$ ), whereas the brimonidinecontaining groups experienced all cases of conjunctivitis (BBFC group, 1.8\%; brimonidine group, 3.0\%), dry mouth (BBFC group, 2.7\%; brimonidine group, 2.1\%), and eye allergy (BBFC group, 4.5\%; brimonidine group, 0.9\%). Fiftytwo patients discontinued participation due to non-serious treatment-related AEs (BBFC, $n=25,11.3 \%$; brinzolamide, $n=5,2.1 \%$; brimonidine, $n=22,9.4 \%$ ). Three of the

Table 2. Demographic and Baseline Characteristics

\begin{tabular}{|c|c|c|c|c|}
\hline Demographic/baseline characteristics & Total population $\mathrm{N}=679$ & $B B F C \mathrm{~N}=218$ & Brinzolamide $\mathrm{N}=229$ & Brimonidine $\mathrm{N}=232$ \\
\hline \multicolumn{5}{|l|}{ Age, years } \\
\hline Mean \pm standard deviation & $64.9 \pm 10.4$ & $65.7 \pm 10.3$ & $64.2 \pm 10.3$ & $64.9 \pm 10.5$ \\
\hline$<65, n(\%)$ & $323(47.6 \%)$ & $98(45.0 \%)$ & $110(48.0 \%)$ & $115(49.6 \%)$ \\
\hline$\geq 65, n(\%)$ & $356(52.4 \%)$ & $120(55.0 \%)$ & $119(52.0 \%)$ & $117(50.4 \%)$ \\
\hline \multicolumn{5}{|l|}{ Race, $n(\%)$} \\
\hline White & $529(77.9 \%)$ & $174(79.8 \%)$ & $179(78.2 \%)$ & $176(75.9 \%)$ \\
\hline Black & $130(19.1 \%)$ & $36(16.5 \%)$ & $42(18.3 \%)$ & $52(22.4 \%)$ \\
\hline Asian & $9(1.3 \%)$ & $3(1.4 \%)$ & $5(2.2 \%)$ & $1(0.4 \%)$ \\
\hline Multi-racial & $3(0.4 \%)$ & $0(0 \%)$ & $1(0.4 \%)$ & $2(0.9 \%)$ \\
\hline Other & $8(1.2 \%)$ & $5(2.3 \%)$ & $2(0.9 \%)$ & $1(0.4 \%)$ \\
\hline \multicolumn{5}{|l|}{ Sex, $n(\%)$} \\
\hline Male & $298(43.9 \%)$ & $100(45.9 \%)$ & $97(42.4 \%)$ & $101(43.5 \%)$ \\
\hline Female & $381(56.1 \%)$ & $118(54.1 \%)$ & $132(57.6 \%)$ & $131(56.5 \%)$ \\
\hline \multicolumn{5}{|l|}{ Diagnosis, $n$ (\%) } \\
\hline Ocular hypertension & $168(24.7 \%)$ & $51(23.4 \%)$ & $59(25.8 \%)$ & $58(25.0 \%)$ \\
\hline Open-angle glaucoma & $511(75.3 \%)$ & $167(76.6 \%)$ & $170(74.2 \%)$ & $174(75.0 \%)$ \\
\hline
\end{tabular}

Demographics and baseline characteristics were presented from the intent-to-treat population. Intraocular pressure was analyzed using the intent-to-treat population. $\mathrm{BBFC}$, brinzolamide $1 \%$ /brimonidine $0.2 \%$ fixed combination. 


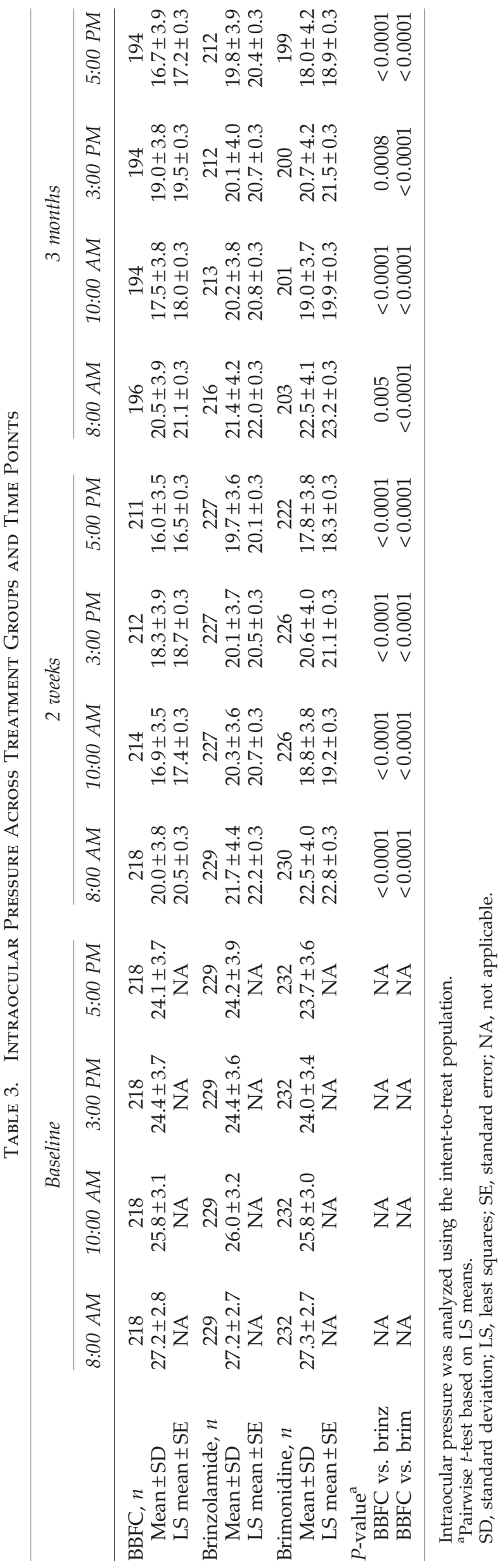

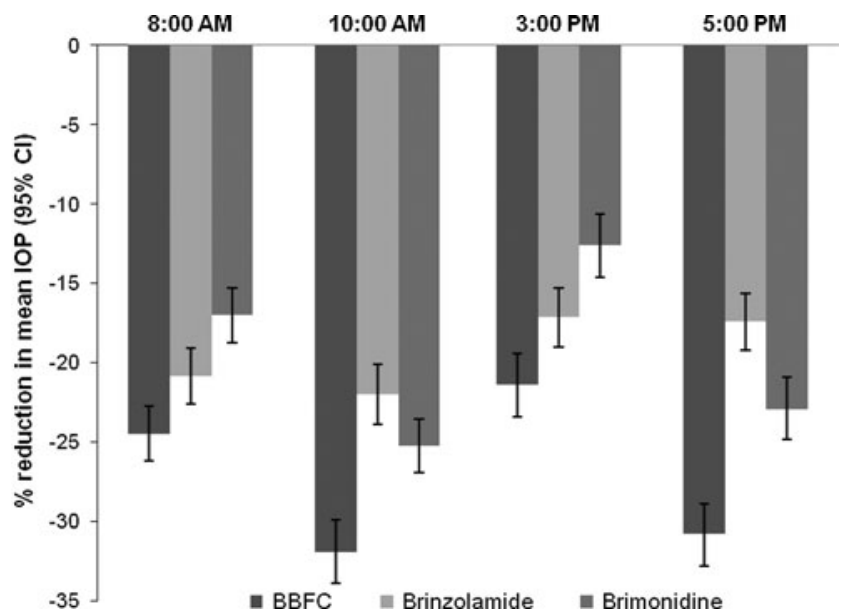

FIG. 1. Percent reduction in mean intraocular pressure (IOP) from the baseline visit to the 3-month visit across treatment groups and time points.

treatment-related AEs were severe (allergic conjunctivitis, $n=1$, BBFC group; blurred vision, $n=1$, brinzolamide group; and atopic dermatitis, $n=1$, brimonidine group). The allergic conjunctivitis and atopic dermatitis resolved with treatment, and the blurred vision resolved without treatment. Ten patients experienced nonfatal serious AEs (BBFC group, $n=4$, $1.8 \%$; brinzolamide, $n=1,0.4 \%$; brimonidine, $n=5,2.1 \%$ ), none of which were judged to be related to treatment.

From the baseline visit to the 3-month visit, the change in mean number of letters read was $<1$ letter in all groups. Using slit-lamp biomicroscopy, investigators observed $\geq 1$ unit increases from the baseline visit to the exit visit (last ontherapy visit up to and including 3-month visit) for eyelids / conjunctiva in $12.7 \%$ (28 of 221 ) of the BBFC group, $3.0 \%$ (7 of 232) of the brinzolamide group, and 9.5\% (22 of 234) of the brimonidine group. No other significant changes were noted in visual acuity, anterior or posterior segment examination, pachymetry or perimetry.

A slight trend toward a decrease in both systolic and diastolic mean blood pressure was observed from the baseline visit to the 3-month visit at the 10:00 AM time point for patients from the BBFC group (4.4 mm Hg systolic decrease and $2.3 \mathrm{~mm} \mathrm{Hg}$ diastolic decrease) and the brimonidine group (5.0 mm Hg systolic decrease and $2.4 \mathrm{~mm} \mathrm{Hg}$ diastolic decrease), but the scatter plots in Fig. 2 show that individual patients' blood pressure remained relatively stable from baseline to 3 months, regardless of the study medication used. One patient from the BBFC group had a blood pressure decrease coded as an AE. No patient experienced a clinically meaningful decrease in pulse rate.

\section{Discussion}

In the current study, the BBFC group demonstrated significantly lower mean IOPs than either the brinzolamide group $(P \leq 0.01)$ or the brimonidine group $(P<0.0001)$ across all 4 time points and across all visits, starting at 2 weeks after treatment initiation and continuing through 3 months. In addition, fewer patients in the BBFC group discontinued the study due to a lack of IOP control $(0.5 \%)$ than did patients from either of the monotherapy groups $(3.0 \%$, brinzolamide; $5.5 \%$, brimonidine). Taken together, these observations demonstrate that the IOP-lowering contribution of the combination therapy is greater than the contribution of 
Table 4. Treatment-Related Adverse Events with an Incidence $\geq 1 \%$

\begin{tabular}{|c|c|c|c|}
\hline Treatment-related adverse event & $B B F C \mathrm{~N}=221 \mathrm{n}(\%)$ & Brinzolamide $\mathrm{N}=234 \mathrm{n}(\%)$ & Brimonidine $\mathrm{N}=235 \mathrm{n}(\%)$ \\
\hline \multicolumn{4}{|l|}{ Ocular } \\
\hline Vision blurred & $10(4.5 \%)$ & $16(6.8 \%)$ & $0(0 \%)$ \\
\hline Eye irritation & $12(5.4 \%)$ & $4(1.7 \%)$ & $6(2.6 \%)$ \\
\hline Eye allergy & $10(4.5 \%)$ & $0(0 \%)$ & $2(0.9 \%)$ \\
\hline Eye pain & $6(2.7 \%)$ & $4(1.7 \%)$ & $3(1.3 \%)$ \\
\hline Eye pruritus & $5(2.3 \%)$ & $3(1.3 \%)$ & $0(0 \%)$ \\
\hline Conjunctivitis & $4(1.8 \%)$ & $0(0 \%)$ & $7(3.0 \%)$ \\
\hline Conjunctivitis allergic & $4(1.8 \%)$ & $1(0.4 \%)$ & $5(2.1 \%)$ \\
\hline Conjunctival hyperemia & $4(1.8 \%)$ & $1(0.4 \%)$ & $2(0.9 \%)$ \\
\hline Dry eye & $4(1.8 \%)$ & $2(0.9 \%)$ & $1(0.4 \%)$ \\
\hline Lacrimation increased & $3(1.4 \%)$ & $1(0.4 \%)$ & $1(0.4 \%)$ \\
\hline Ocular hyperemia & $2(0.9 \%)$ & $1(0.4 \%)$ & $6(2.6 \%)$ \\
\hline Conjunctival follicles & $1(0.5 \%)$ & $0(0 \%)$ & $3(1.3 \%)$ \\
\hline \multicolumn{4}{|l|}{ Non-ocular } \\
\hline Dysgeusia & $9(4.1 \%)$ & $24(10.3 \%)$ & $1(0.4 \%)$ \\
\hline Dry mouth & $6(2.7 \%)$ & $0(0 \%)$ & $5(2.1 \%)$ \\
\hline Fatigue & $1(0.5 \%)$ & $0(0 \%)$ & $4(1.7 \%)$ \\
\hline
\end{tabular}

Adverse events were analyzed using the safety population.

either of its components. Moreover, they demonstrate that this effect occurs early in the treatment course and is maintained through 3 months of treatment. The magnitude of IOP reductions from baseline at 3 months observed in the current study with brinzolamide $1 \%(4.2-5.7 \mathrm{~mm} \mathrm{Hg})$ and brimonidine $0.2 \% \quad(3.1-6.5 \mathrm{~mm} \mathrm{Hg})$ are consistent with reductions previously reported from phase 3 trials of brinzolamide TID (4.1-5.6 mm Hg) $)^{13,14}$ and brimonidine TID (3.1-6.3 $\mathrm{mm} \mathrm{Hg}),{ }^{15,16}$ dispelling the possibility that the superiority of the BBFC IOP reductions (5.4-8.4 mm Hg) could
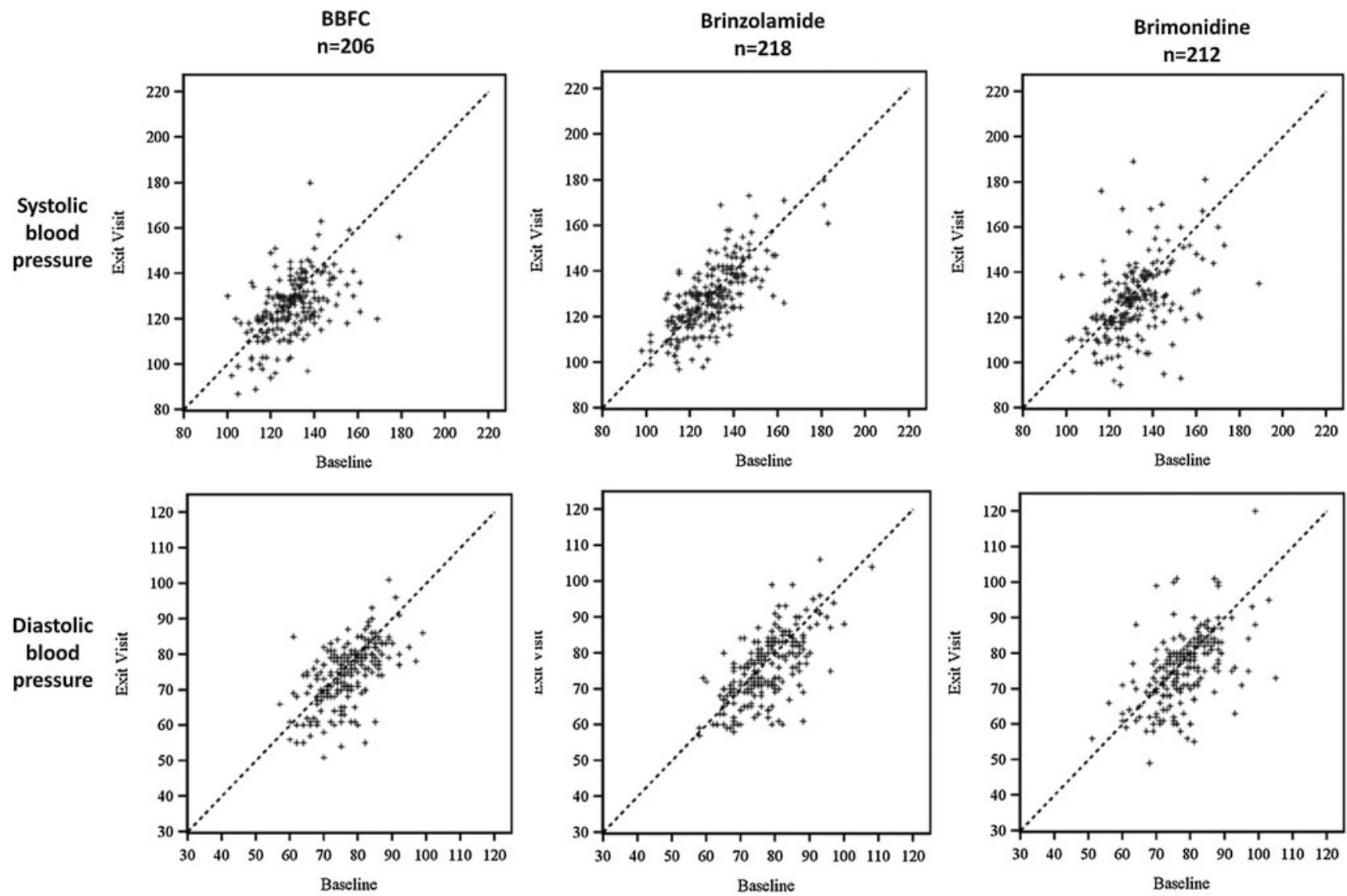

$\mathrm{BBFC}=$ brinzolamide/brimonidine fixed combination Blood pressure was analyzed using the safety population.

FIG. 2. Distribution of systolic and diastolic blood pressures at 10:00 AM: baseline visit versus exit visit. 
be explained by inferior performance of the individual monotherapies.

BBFC provided consistent diurnal IOP control. IOP was significantly lower from baseline, and lower than in either monotherapy group, at every visit and every time point in the BBFC group $(P \leq 0.01)$. These results suggest that a nonbeta blocker, non-PGA fixed combination can provide effective IOP control.

The BBFC group exhibited safety findings that are consistent with those associated with the individual components. Treatment-related AEs occurring in the BBFC group $(26.2 \%)$ were slightly higher than the $18.8 \%$ of AEs in the brinzolamide group and $17.4 \%$ in the brimonidine group, and those treatment-related AEs leading to discontinuation were also higher in the BBFC group (11.3\%) compared with the brinzolamide group $(2.1 \%)$ and the brimonidine group $(9.4 \%)$. However, no serious treatment-related AEs were observed in the BBFC group, nor were any unexpected safety signals observed in this study. Cardiovascular parameters (resting pulse rate and systolic and diastolic mean blood pressure) in the BBFC group were similar to those in the brimonidine group, demonstrating a modest, clinically insignificant decline.

Different dosing schedules of some IOP-lowering agents (single entities and fixed-combination therapies) are sometimes approved inside and outside the United States. The dosing schedule of BBFC in this study is consistent with the approved dosing schedules of the individual components by the U.S. Food and Drug Administration. BBFC is currently being tested outside the United States with twice daily (BID) dosing to assess its IOP-lowering ability. ${ }^{17}$

This clinical trial had some limitations. The short, 3-month design of the study prevents examination of the long-term effects of BBFC on the IOPs of patients with open-angle glaucoma or ocular hypertension. However, additional safety data will be collected through the 6-month visit, allowing analysis of the safety of BBFC to extend to 6 months. Further, the comparison of BBFC with its individual components does not provide any insight as to how this fixed-combination therapy might be compared with other fixed-combination therapies or when administered adjunctively to other glaucoma agents in a different class. Further studies are needed to address these limitations.

In conclusion, this study demonstrated that BBFC has significantly greater antihypertensive activity compared with either brinzolamide $1 \%$ monotherapy or brimonidine $0.2 \%$ monotherapy in patients with open-angle glaucoma or ocular hypertension, and it has a safety profile which is consistent with that of its individual components.

\section{Acknowledgment}

Jennifer Klem, PhD, provided medical writing assistance, which was funded by Alcon Research, Ltd.

\section{Author Disclosure Statement}

Both Nguyen and Whitson are on the speaker's bureaus for Alcon, Allergan, and Merck. Goode is a consultant to Alcon. Realini is a consultant to Alcon and is on the speaker's bureau for Lumenis. McMenemy is a consultant to Alcon and Bausch \& Lomb; is on the speaker's bureaus for Alcon, Allergan, Bausch \& Lomb, and Merck; and receives research funding from Abbott, Alcon, and Sunpharma.
The authors have no proprietary interest in the materials presented herein.

\section{References}

1. The Advanced Glaucoma Intervention Study (AGIS): 7. The relationship between control of intraocular pressure and visual field deterioration. The AGIS Investigators. Am. J. Ophthalmol. 130:429-440, 2000.

2. Leske, M.C., Heijl, A., Hussein, M., et al. Factors for glaucoma progression and the effect of treatment: the early manifest glaucoma trial. Arch. Ophthalmol. 121:48-56, 2003.

3. Musch, D.C., Gillespie, B.W., Niziol, L.M., Lichter, P.R., and Varma, R. Intraocular pressure control and long-term visual field loss in the Collaborative Initial Glaucoma Treatment Study. Ophthalmology 118:1766-1773, 2011.

4. Kass, M.A., Heuer, D.K., Higginbotham, E.J., et al. The Ocular Hypertension Treatment Study: a randomized trial determines that topical ocular hypotensive medication delays or prevents the onset of primary open-angle glaucoma. Arch. Ophthalmol. 120:701-713, 2002.

5. Kass, M.A., Gordon, M.O., Gao, F., et al. Delaying treatment of ocular hypertension: the ocular hypertension treatment study. Arch. Ophthalmol. 128:276-287, 2010.

6. Chrai, S.S., Makoid, M.C., Eriksen, S.P., and Robinson, J.R. Drop size and initial dosing frequency problems of topically applied ophthalmic drugs. J. Pharm. Sci. 63:333-338, 1974.

7. Jaenen, N., Baudouin, C., Pouliquen, P., et al. Ocular symptoms and signs with preserved and preservative-free glaucoma medications. Eur. J. Ophthalmol. 17:341-349, 2007.

8. Pisella, P.J., Pouliquen, P., and Baudouin, C. Prevalence of ocular symptoms and signs with preserved and preservative free glaucoma medication. Br. J. Ophthalmol. 86:418-423, 2002.

9. Timolol maleate ophthalmic solution [package insert]. Fort Worth, TX: Falcon Pharmaceuticals, Ltd.; 2004.

10. Bournias, T.E., and Lai, J. Brimonidine tartrate $0.15 \%$, dorzolamide hydrochloride $2 \%$, and brinzolamide $1 \%$ compared as adjunctive therapy to prostaglandin analogs. Ophthalmology 116:1719-1724, 2009.

11. Day, D.G., and Hollander, D.A. Brimonidine purite $0.1 \%$ versus brinzolamide $1 \%$ as adjunctive therapy to latanoprost in patients with glaucoma or ocular hypertension. Curr. Med. Res. Opin. 24:1435-1442, 2008.

12. Reis, R., Queiroz, C.F., Santos, L.C., Avila, M.P., and Magacho, L. A randomized, investigator-masked, 4-week study comparing timolol maleate $0.5 \%$, brinzolamide $1 \%$, and brimonidine tartrate $0.2 \%$ as adjunctive therapies to travoprost $0.004 \%$ in adults with primary open-angle glaucoma or ocular hypertension. Clin. Ther. 28:552-559, 2006.

13. Silver, L.H. Clinical efficacy and safety of brinzolamide (Azopt), a new topical carbonic anhydrase inhibitor for primary open-angle glaucoma and ocular hypertension. Brinzolamide Primary Therapy Study Group. Am. J. Ophthalmol. 126:400-408, 1998.

14. Sall, K. The efficacy and safety of brinzolamide 1\% ophthalmic suspension (Azopt) as a primary therapy in patients with open-angle glaucoma or ocular hypertension. Brinzolamide Primary Therapy Study Group. Surv. Ophthalmol. 44 Suppl 2:S155-S162, 2000.

15. Craven, E.R., Walters, T.R., Williams, R., et al. Brimonidine and timolol fixed-combination therapy versus monotherapy: a 3-month randomized trial in patients with glaucoma or ocular hypertension. J. Ocul. Pharmacol. Ther. 21:337-348, 2005. 
16. Stewart, W.C., Sharpe, E.D., Harbin, T.S., Jr., et al. Brimonidine $0.2 \%$ versus dorzolamide $2 \%$ each given three times daily to reduce intraocular pressure. Am. J. Ophthalmol. 129:723-727, 2000.

17. ClinicalTrials.gov website. Brinzolamide/brimonidine twice a day (BID) fixed combination (FC) vs. brinzolamide BID and brimonidine BID in patients with open angle glaucoma or ocular hypertension. Available at: http:// clinicaltrials.gov/ct2/show/NCT01310777. Accessed July 9, 2012.
Received: October 25, 2012

Accepted: January 04, 2013

Address correspondence to: Dr. Quang H. Nguyen Scripps Clinic 10666 North Torrey Pines Road, MS 214 La Jolla, CA 92037

E-mail: nguyen.quang@scrippshealth.org 